\title{
The Significant Impact of Aromatherapy, Medical Food, Exercise Training, Music with Movement, Home Environment, Caregiver Characteristics, and Occupational Therapy on Alzheimer Disease
}

\author{
Hassan Izzeddin Sarsak* \\ Department of Occupational Therapy, Batterjee Medical College, Saudi Arabia \\ *Corresponding author: Hassan Izzeddin Sarsak, Department of Occupational Therapy, Batterjee Medical College, Jeddah, KSA, Saudi Arabia, \\ Email: sarsakhassan@gmail.com
}

Submission: 制 October 10, 2018; Published: 阱 October 29, 2018

\begin{abstract}
The main purpose of this paper was to provide an overview and summarize evidence of the impact of different factors and evidence-based interventions on symptoms management and functional performance and cognitive function for patients with Alzheimer disease including but not limited to occupational therapy interventions.

Aromatherapy, medical food, exercise training, music with movement, home environment modifications, caregiver characteristics, and occupational therapy are all important significant factors and evidence-based interventions that healthcare practitioners need to take into consideration and involve into the interdisciplinary and comprehensive evaluation and treatment plans for patients with Alzheimer Disease.

Keywords: Alzheimer's disease; Dementia; Nutritional intervention; Group music with movement; Agitated behaviors; Institutionalized elders; Nursing home; Physical activity; Randomized controlled trial; Disability; Activities of daily living; Occupational therapy
\end{abstract}

\section{Introduction}

Alzheimer Disease (AD) is a progressive, degenerative disease of the cerebral cortex resulting in diffuse atrophy of the cerebral cortex. The disease is characterized by neurofibrillary tangles, betaamyloid plaques, and neuronal degeneration [1]. Today there are an estimated 5.3 million Americans with Alzheimer's disease. This number is expected to triple by 2050 . Dementia is a serious public health problem currently affecting 18 million people worldwide [2]. Early symptoms of AD include: frequently repeating statements, misplacing items, difficulty finding names for familiar objects, getting lost on familiar routes and changes in personality and emotional responses. In the middle stages, those with $\mathrm{AD}$ forget basic information about the world, such as how to use common objects or how to get from point to another. In the later stages, skills like dressing; bathing and reading become more difficult. In the final stages of Alzheimer's, the person returns to the functioning of an infant [3].

The Cognitive Performance Test (CPT) was developed as a research instrument to provide a baseline measure of function in individuals with $\mathrm{AD}$ and to track change over time. Currently, the test is used in clinics, long term care and in the home. The test uses seven common daily living tasks (medication management, shopping, using a map, making a phone call, getting dressed, making toast and washing hands). A gross level score is obtained for each task; these scores are then added and averaged to determine the cognitive level [2].
The main purpose of this review article was to provide an overview and summarize evidence of the impact of different factors and evidence-based interventions on symptoms management and functional performance and cognitive function for patients with Alzheimer disease including but not limited to occupational therapy interventions.

This paper is organized as follows: section 2 presents the impact of aromatherapy in sleep promotion in AD, section 3 introduces the impact of medical food on cognitive function in people with mild $A D$, section 4 discusses the impact of exercise program on cognitive function in people with mild $\mathrm{AD}$, section 5 explains the impact of group music with movement on agitated behaviours in people with mild AD, section 6 summarizes comparing two programs of cognitive training in $\mathrm{AD}$, section 7 introduces the impact of home environmental intervention on self-efficacy and upset in caregivers and daily function of dementia patients, sections 8 highlights the role of occupational therapy in dementia, and finally section 9 sheds light on conclusions and some clinical recommendations.

Impact of aromatherapy in sleep promotion in Alzheimer's disease (AD)

Researchers conducted a pilot study to examine the feasibility of administering a warm lavender oil towel bath in the homes of patients with dementia who experienced sleep problems suggesting 
that lavender may help people with dementia fall asleep faster [1].

The sample includes six people with dementia and six caregivers bathed the patients with warm towels moistened with a mixture of lavender oil and a no-rinse soap solution for a period of 10 minutes for 7 nights. The amount of time for participants to fall asleep (sleep-onset latency) decreased on average from $5.51 \mathrm{~min}$ utes $(\mathrm{SD}=7.98)$ to 2.78 minutes $(\mathrm{SD}=4.92 ; \mathrm{P}=0.06)$ [1].

Daytime napping decreased consistently; however, night-time awakenings remained unchanged. Caregivers reported, for example, significant decreases in patients with dementia waking up too early and a reduction in the severity of difficulty falling asleep and staying asleep. Larger, clinical trials are needed [1].

\section{Impact of medical food on cognitive function in people with mild Alzheimer's disease (AD)}

Randomized controlled trial (RCT) was used with 225 AD patients participated in this randomized, double-blind controlled trial [4]. The primary objective of this double-blind, randomized, controlled, multicentre trial was to determine the effect of a medical food (Product ID 4804/4805) on cognitive function compared with a control product in patients with mild AD, after a 12-week supplementation. Secondary objectives were to assess its effects on safety, tolerability and compliance, behaviour, functional abilities, quality of life, biochemical parameters, and cognitive performance after 12 and 24 weeks of supplementation [4].

Patients received the active or control product as a drink (125mL tetra packages), available in two flavours, to be taken each day at breakfast, and consumed within 1 hour. Primary outcome measures were the delayed verbal recall task of the Wechsler Memory Scale-revised, and the 13-item modified Alzheimer's Disease Assessment Scale-cognitive subscale. At week 12, significant improvement in the delayed verbal recall task was noted in the active group compared with control $(\mathrm{P}=0.021)$. Supplementation with a medical food including phosphatide precursors and cofactors for 12 weeks improved memory (delayed verbal recall) in mild AD patients [4].

\section{Impact of exercise program on cognitive function in peo- ple with mild Alzheimer's disease (AD)}

This study was a 12-month multicentre, randomized, controlled, single-blind study of parallel groups of 134 ambulatory subjects with AD living in five nursing homes in Toulouse, France. It studied the impact of an exercise program in improving ability to perform activities of daily living (ADLs), physical performance, nutritional status and decreasing behavioural disturbance and depression in patients with Alzheimer's disease (AD) [5].

Collective exercise program (1 hour, twice weekly of walk, strength, balance, and flexibility training) or routine medical care for 12 months were applied. Primary outcome measure; the primary effectiveness was decline from baseline in Katz ADL score 23 of exercise participants compared with decline in ADL score of the control group at 12 months of follow-up. The Katz Index of ADLs 23 was used to address the question of whether exercise may prevent the worsening of disabilities in nursing home residents suffering from AD. Secondary outcome measures; the secondary effectiveness outcomes included ADL score at 6 months, measures of physical performance, nutritional status, behavioural disturbance and depression [5].

This study provides evidence that a moderate exercise program conducted twice a week significantly slows, by approximately onethird, the progressive deterioration in ability to perform ADLs in people with $\mathrm{AD}$ living in nursing homes $(\mathrm{P}=0.02)$. A simple exercise program, 1 hour twice a week, led to significantly slower decline in ADL score in patients with AD living in a nursing home than routine medical care [5].

\section{Impact of group music with movement on agitated be- haviours in people with mild Alzheimer's disease (AD)}

Agitated behaviours are identified by caregivers as the most challenging in dementia care. Alternative approaches reducing occurrence of agitated behaviours and the need for chemical or physical restraints become valuable for institutionalized elders with dementia [6].

A randomized controlled trial was used. 36 institutionalized elders with dementia completed the study with 18 in the experimental group receiving group music with movement intervention was administered $30 \mathrm{~min}$ in afternoon twice a week for 4 weeks and 18 in the control group receiving usual care without intervention [6].

Increased use of music as a therapeutic intervention may reflect the growing interest in complementary therapies in health care in the last decade. The therapeutic aims for use of music are to bring about positive patient outcomes and to aid in physiological, psychological and emotional integration of a person during treatment of an illness or disability. Music can change the reactions of autonomic nervous system in the thalamus and regulate the nervous impulse conductions of the limbic system and reticular activating system [6].

Agitated behaviours were significantly reduced in the experimental group following 4 weeks of group music with movement intervention compared to that of the control group $(p<0.001)$. Group music with movement intervention can be beneficial in managing agitated behaviours of those with dementia and should be incorporated into care routines in residential facilities [6].

\section{Comparing two programs of cognitive training in Alzhei- mer's disease}

Pilot study was used with $22 \mathrm{AD}$ patients to evaluate the efficacy of two different procedures of individual cognitive training in mild to moderate Alzheimer's disease. They compared stimulation of procedural memory (group 1) with training of partially spared cognitive functions (group 2) [7].

Different neuropsychological functions are not equally affected in $\mathrm{AD}$, in the most cases episode memory is first affected, followed 
only later by semantic memory and instrumental functions, and later on by executive functions. Data also demonstrate a relative sparing of procedural memory and perspective priming in contrast with declarative memory. Several manipulations aimed to structure the acquisition and retrieval of memory tracts have shown to improve learning in $\mathrm{AD}$ [7].

In this pilot study, assessment included: neuropsychological tests, and the Functional Living Skills Assessment (FLSA), a standardized battery built to directly evaluate patients' performance in everyday life. They observed a significant improvement for both groups after training in FLSA total score $(\mathrm{P}=0.005)$ and subscales. For group 1, they also found a slightly improved performance in two tests: Attentional Matrices $(\mathrm{P}=0.041)$, and Verbal Fluency for Letters $(\mathrm{P}=0.059)$. After 3 months, patients' results showed a tendency to regress to the pre-training level. Both AD groups showed a substantial improvement after training in a direct performance measure of everyday functioning. However, results at neuropsychological tests suggested that training activities of daily living (supported by procedural memory) may be more effective than stimulating residual cognitive functions [7].

\section{Impact of home environmental intervention on self-ef- ficacy and upset in caregivers and daily function of de- mentia patients}

A randomized controlled trial was used. They determined short-term effects of a home environmental intervention on self-efficacy and upset in caregivers and daily function of dementia patients. They also determined if treatment effect varied by caregiver gender, race, and relationship to patient. Families ( $N=171)$ of dementia patients were randomized to intervention or usual care control group. The intervention involved 5 90-min home visits by occupational therapists who provided education and physical and social environmental modifications. Compared with controls, intervention caregivers reported fewer declines in patients instrumental activities of daily living (IADL) $(\mathrm{P}=0.030)$ and less decline in self-care and fewer behaviour problems in patients at 3 months post-test. Also, intervention spouses reported reduced upset $(\mathrm{P}=0.049)$, women reported enhanced self-efficacy in managing behaviours $(\mathrm{P}=0.038)$, and women $(\mathrm{P}=0.049)$ and minorities $(\mathrm{P}=0.037)$ reported enhanced self-efficacy in managing functional dependency. The environmental program appeared to have a modest effect on dementia patients' IADL dependence. Also, among certain subgroups of caregivers the program improved self-efficacy and reduced upset in specific areas of caregiving [8].

\section{Role of occupational therapy}

Occupational therapy (OT) practitioners help those with AD and their caregivers to live life to its fullest. This is done through adapting to the environment and focusing on what they can do to maximize engagement in activity, promote safety and enhance quality of life [2]. Enhancing function, promoting social participation, and finding ways for individuals with dementia to have a meaningful and enjoyable life are the keys to successful occupational therapy intervention. Occupational therapists play an important role in the evaluation and intervention process for persons with dementia and can help in so many ways through the application of a variety of therapeutic approaches that help improve function through compensation or adaptation, develop coping skills for caregivers, and ensure safe and supportive modified environment [3].

\section{Conclusion and Recommendation}

Aromatherapy, medical food, exercise training, music with movement, home environment modifications, caregiver characteristics, and occupational therapy are all important factors and evidence-based interventions that healthcare practitioners need to take into consideration and involve into the interdisciplinary and comprehensive evaluation and treatment plans for patients with Alzheimer Disease.

Furthermore, this review suggests some recommendations. Firstly, a general neurological and physical examination should be performed on all patients presenting with dementia. Secondly, all guidelines should emphasize the importance of educating patients and their families about the disease process and its expected course. Thirdly, early referral to local support groups is recommended, and medicolegal issues such as driving, and end-of-life planning should be addressed. Fourthly, the decision to treat with medication should be shared with the patient and caregivers, including a discussion of the modest clinical benefit, adverse effects, and cost. Finally, physicians should consider discontinuing therapy in patients who continue to decline despite maximal therapy modify treatment plan, make recommendations, and follow up accordingly as needed.

\section{References}

1. Uriri Glover J, McCarthy M, Cesarotti E (2012) Solving the puzzle of Alzheimer disease. Nurse Pract 37(9): 21-27.

2. Waverly Health Center (2012) Occupational therapy and dementia/Alzheimer's, p. 39.

3. Sarsak HI (2018) Overview: Dementia and the role of occupational therapy practitioner. MOJ Yoga Physical Ther 3(5): 69-75.

4. Scheltens P, Kamphuis PJ, Verhey FR, Olde MG, Wurtman RJ, et al. (2010) Efficacy of a medical food in mild Alzheimer's disease: A randomized, controlled trial. Alzheimer's \& Dementia 6(1): 1-10.

5. Rolland Y, Pillard F, Klapouszczak A, Reynish E, Thomas D, et al. (2007) Exercise program for nursing home residents with alzheimer's disease: A 1-Year randomized, controlled trial. The American Geriatrics Society 55 (2): 158-165.

6. Sung HC, Chang SM, Lee WL, Lee MS (2006) The effects of group music with movement intervention on agitated behaviors of institutionalized elders with dementia in Taiwan. Complement Ther Med 14(2): 113-119.

7. Fioravanti R, Chiavari L, Imbornone E, Alberoni M, Pomati S, et al. (2002) Comparing two programs of cognitive training in alzheimer's disease: A pilot study. Acta Neurologica Scandinavica 105: 365-371.

8. Gitlin LN, Corcoran M, Winter L, Boyce A, Hauck W (2001) A Randomized, controlled trial of a home environnent mental intervention: Effect on efficacy and upset in caregivers and on daily function of persons with dementia. Gerontologist 41 (1): 4-14. 
(c) (i) Creative Commons Attribution 4.0 International License

For possible submissions Click Here
PPRS

Psychology and Pyychotherespy: -

Submit Article

\section{Psychol Psychother Res Stud}

\section{Benefits of Publishing with us}

- High-level peer review and editorial services

- Freely accessible online immediately upon publication

- Authors retain the copyright to their work

- Licensing it under a Creative Commons license

- Visibility through different online platforms 